\title{
The Photoacoustic Spectroscopy Applied in the Characterization of the Cross-linking Process in Polymeric Materials
}

\author{
D. T. Dias, A. N. Medina, M. L. Baesso, A. C. Bento*, \\ Universidade Estadual de Maringá, Departamento de Física \\ Av. Colombo 5790, 87020-900, Maringá - Paraná, Brazil \\ M. F. Porto, and A. F. Rubira \\ Universidade Estadual de Maringá, Departamento de Química \\ Av. Colombo 5790, 87020-900, Maringá - Paraná, Brazil
}

Received on 6 November, 2001

\begin{abstract}
In this work we used the Photoacoustic Spectroscopy (PAS) to evaluate the crosslinking of the copolymer from ethylene vinyl trimethoxysilane (EVS) and the grafted vinyl trimethoxysilane (VTS) on low density polyethylene (LDPE). PAS is used for sounding the overtone bands and stretching frequencies combinations of the groupings $-\mathrm{Si}-\mathrm{OH},=\mathrm{CH}_{2},-\mathrm{CH}_{3}$ and $-\mathrm{CH}_{2}-\mathrm{CH}_{3}$, in the near and medium infrared range. The samples were typically prepared with $3 \%, 5 \%$ and $7 \%$ of catalyst and crosslinked in the temperatures of 70,80 and $90^{\circ} \mathrm{C}$. Using the overtone bands of $-\mathrm{OH}$ groups the PAS shows the optimum combination, pointing a better crosslinking effect for $80^{\circ} \mathrm{C}$ and in the range $5 \%$ to $7 \%$ of catalyst, typically.
\end{abstract}

\section{Introduction}

The Photoacoustic Spectroscopy (PAS) is usually used for a nondestructive analysis of a variety of materials in the visible and infrared range $[1,2]$. As it is a spectroscopic technique, it is expected that identifying the absorption bands in the cross-linking process can follow the cross-linking process. Although the crosslinking process could be monitored using Fourier transform technique (FTIR) in the far infrared range (10000 to $40000 \mathrm{~cm}^{-1}$ ), by using the PAS technique it is made easier to analyze the process in the near infrared (NIR, 800 to $1600 \mathrm{~nm}$ ) and medium infrared (MIR, 1600 to $3200 \mathrm{~nm}$ ) because of the overtones of hydroxyl bonds and their combination with stretching modes of $\mathrm{CH}$ bonds that are found either more defined or separated in this spectral range[3, 4].

Nowadays, polymeric materials have been largely utilized in industries for producing a wide range of special goods, each one for a specific purpose of application. It is concerned that medium voltage application is a big deal for both the grafted PE and ethylene vinyltrimethoxysilane (EVS) due to the needs of a good thermal conductivity and high electric rigidity. The reduction of the electric rigidity occurs after crystalline phase is broken up either during fabric process or after the extreme conditions of usage like in the hostile environment like that found in power delivery wiring [5].
One of most utilized polymer is polyethylene (PE) that should be modified to achieve the electric requirements to be used as insulating material. Aside thermoplastic and rubber cross-linked, PE is the worldwide used as electrical insulating wires, especially in applications that need high temperature operation. The physical properties of cross-linked PE can be enhanced in this process and they will be dependent on the cross-linking rate [6]. Usually, the PE cross-linked by means of Sioplas technology [7], is found mostly in the low voltage wiring, because it comprises a good heat transfer allied to the low electrical conductivity.

In this paper we present the PAS study of the crosslinking process in the grafted $\mathrm{PE}$ and for the Copolymer EVS. The overtones band are followed for a set of samples that were prepared using mainly two free variables, catalyst concentration and temperature of water vapor, utilized for cross-linking the polymers.

\section{Photoacoustic Method}

The spectral range of our interest here is 700 to 2600 $\mathrm{nm}$ corresponding to NIR and MIR region, where the absorption bands are mainly due to overtones and combination of the fundamental modes of vibration and rotational bands. The many possible combination such 
as stretching, bending, scissoring, wagging make it difficult to assign the fundamental absorption.

The molecules present a harmonic behavior for highenergy vibrational states and present a tendency of dissociation if the bond has a high potential energy. In this case the vibrational state no longer can behaves as a harmonic oscillator and anharmonic state takes place.

It can be shown that for an anharmonic oscillator the energy is given by $[8,9,10]$ :

$$
E=h v_{o s c}(\eta+1 / 2)-h v_{o s c} x_{e}(\eta+1 / 2)^{2}+h v_{o s c} y_{e}(\eta+1 / 2)^{3}+\ldots
$$

here $x_{e}$ and $y_{e}$ are anharmonic constants, $\eta$ is the quantum level index, $v_{\text {osc }}=\left(c / \lambda_{0}\right)$ is the oscilattor frequency, being $c$ the speed of light and $\lambda_{0}$ is the fundamental absorption peak in $\mathrm{nm}$ or $\mathrm{cm}^{-1}$.

The most remarkable feature of such a molecular anharmonic vibration is that transition for more then one energy level is allowed. The overtones transition rates is increased and it reduces the transition probability for higher energy bands, so the $1^{\text {st }}$ overtone is weaker and so it will be the $2^{\text {nd }}$ one if they are compared to the fundamental absorption. The overtone may occur at wavelength between $\lambda_{0} / 2$ and $\lambda_{0} / 3$ and thus the overtones for a specific molecule do not occur at the same spectral region.

The near infrared region is dominated by stretching modes of $O-H, N-H$, and $C-H$ bond in both ways as overtones or as combination with other vibrational types. The overtone bands can be better isolated in the NIR and MIR region due to the anharmonic constant variation or better instrumental resolution. If a specific overtone is assigned then it can be taken for the analysis.

By means of the PAS method, in the region 1050$2600 \mathrm{~nm}$ we were able to identify and assign the absorption bands for the polyethylene, referring to the overtones and their combination with stretching modes. It was possible to accompanying the cross-linking process of the sample in an indirect way.

The photoacoustic effect consists in generating an acoustic signal inside a tightly closed cell filled with gas. The acoustic signal comes from a sample that transfers heat to the gas after being illuminated by modulated light in a given frequency $\omega$. The heat is transferred into the gas at the same frequency as sample is heated producing then the periodic pressure fluctuation inside the chamber. A sensitive microphone coupled in the cell detects this effect. The actual temperature in the sample surface is given by [1]:

$$
\theta_{F}(0)=\frac{\beta I_{o}}{k_{s} \sigma_{s}^{2}\left(r^{2}-1\right)}\left[\frac{(b+1)(r-1) e^{\sigma_{s} l_{s}}-(b-1)(r+1) e^{-\sigma_{s} l_{s}}+2(b-r) e^{-\beta l_{s}}}{(g+1)(b+1) e^{\sigma_{s} l_{s}}-(g-1)(b-1) e^{-\sigma_{s} l_{s}}}\right]
$$

where

$$
b=\frac{k_{b} a_{b}}{k_{s} a_{s}}, \quad g=\frac{k_{g} a_{g}}{k_{s} a_{s}}, \quad r=(1+j) \frac{\beta}{2 a_{s}}=\frac{\beta}{\sigma_{s}},
$$

$a_{i}$ is a thermal diffusion length $\left(\mathrm{cm}^{-1}\right), \beta$ is the optical absorption coefficient at a wavelength $\left(\mathrm{cm}^{-1}\right)$ with intensity $\mathrm{I}_{0}$, is the thermal conductivity (cal/s.cm. ${ }^{0} \mathrm{C}$ ), and de index "s" stands for sample, b= backing and $\mathrm{g}=$ gas. Equation 2 is obtained by solving the coupled set of diffusion equation for the adjacent medium: gas, sample and sample backing. Rosencwaig and Gersho [1] showed that if boundary conditions are applied to the heat flow and temperature continuity in the interfaces and Eq. (2) can be simplified using the characteristics of the sample after comparing then with those specified for the gas and backing.

The photoacoustic signal is shown to be given by the equation:

$$
S_{F}=\left|\frac{\gamma P_{o} \theta(0)}{\ell_{g} \sigma_{g} T_{o}}\right| e^{j \Phi_{F}}
$$

where $\gamma$ is the specific heat ratio $c_{p} / c_{v}, \sigma_{g}$ is the gas complex diffusion coefficient, $P_{0}$ and $T_{0}$ are the ambient 
pressure and the room temperature, respectively. The instrumental phase is $\phi_{f}$ and the gas column depth is $\ell_{g}$, and $\theta(0)$ is the interface sample-gas temperature.

\section{Experimental}

\section{III.1 Material and the Cross-linking Method}

It is known that both copolymer EVS and grafted $\mathrm{PE}$ are susceptible to cross-linking when they are under vapor condition and also it is known that these cross-linked materials presenting some structural differences. In the silane grafted polyethylene, the vinyl trimethoxysilane is grafted to the polymer chain through the $\mathrm{H}^{*}$ abstraction from main chain, resulting in a $C 2$ bridge bonded to the trimethoxysilane. In the EVS copolymer system the trimethoxysilane were introduced during the polymerization and the trimethoxysilane group is bonded to the main chain through the silicon atom.

In the case of silane cross-linking under water vapor and in the presence of condensation catalyst, the alkoxy group of the silane derivative is converted to silanol group and undergoes a condensation reaction with a hydroxyl in a adjacent chain to form " $S i-O-S i$ " type network. Both, the hydrolyses of alkoxy silane to silanol and their condensation reaction occurs almost instantaneously [11].

The cross-linking mechanism of silane grafted PE and EVS copolymer used here follows that described by Kumar [12] and Hjertberg [13], respectively, (see Fig. 1.) Silane-grafted $\mathrm{PE}$ results in a longer and more mobile network when compared with the EVS copolymer and they have been subject of studies of many researches. The interest is focused in many important factors seeking a better understanding about crosslinking kinetics $[14,15,16]$ and also on the morphological changes induced in the polymer chain $[5,17,18]$.

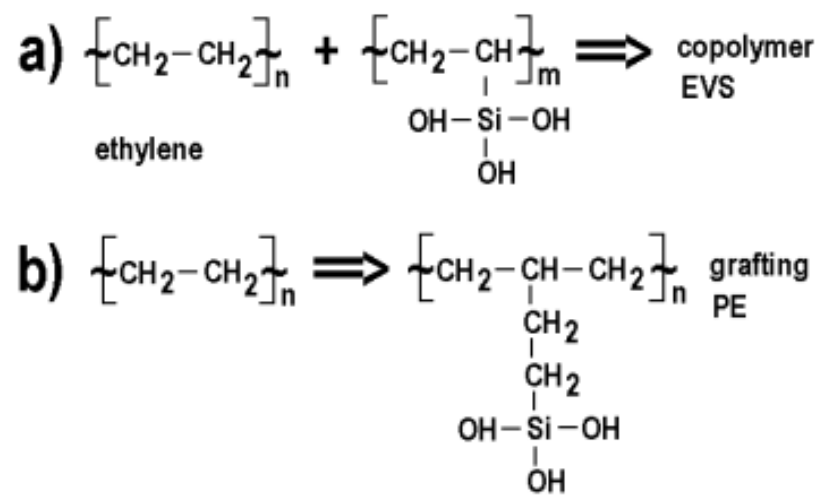

Figure 1. Mechanism of condensation reaction of silanol groups: a) Copolymer EVS and b) Silane grafted PE.

For the present paper we have taken the final condensation reaction as the main base for an indirect spec- troscopic analysis of the cross-linking process in the near and medium infrared range.

\section{III.2 Sample Preparation}

Commercial pellets of copolymer (200 g) were extruded with three different concentration of catalyst that is utilized as cross-linking initiator. The pellets were then milled in a knifed mill suitable for polymers. After this step the pieces were hot pressed in order to perform the copolymer films $(85-595 \mu \mathrm{m}$ thick) and placed in a freezer after being locked in special packing.

The pellets of low density polyethylene (LDPE) (600 g) was kept in a oven at $80^{\circ} \mathrm{C}$ for $12 \mathrm{~h}$ and after, it was grafted with $40 \mathrm{ml}$ of vinyltrimethoxysilane (VTS) whose reaction was initiated by $2.5 \mathrm{~g}$ of benzoyl peroxide by steering the solution during $1 \mathrm{~h}$. The grafted LDPE was then extruded using the same amount of catalyst as in the copolymer EVS described above, keeping also the same steering velocity, torch rate and temperature. The final grafted LDPE, after being grounded, was hot pressed to make films (90-385 $\mu \mathrm{m}$ thick), were maintained in adequate recipients and kept in a freezer before the cross-linking process.

The grafted (LDPE + VTS) polymer (hereafter named as $\mathrm{PE}_{g}$ ) and the copolymer EVS (named as Cop) films were cross-linked in humidity saturated atmosphere in a glass reactor for 8 hours in thermostatic controlled bath at different temperatures. In this way a $3^{2}$ factorial planning design has been applied, the temperature levels were 70,80 and $90^{\circ} \mathrm{C}$ and the catalyst concentration were 3,5 and $7 \%$, in mass. It resulted in nine experiments plus the three base sample, one for each concentration of catalyst.

\section{III.3 Analysis}

Figure 2 shows the home made experimental arrangement for the spectroscopic analysis. It is comprised of an arc lamp of 1000 Watts produced using high pressure Xenon gas (ORIEL 68820). Emitted light is collimated into the inlet slit of the monochromator (ORIEL 77250) which is settled to refract visible light by means of grating in the visible region (ORIEL 77296), near infrared (ORIEL 77299) and medium infrared (ORIEL 77300). They allow one to scan wavelengths from 180 to $800 \mathrm{~nm}, 800$ to $1600 \mathrm{~nm}$ and 1600 to $3200 \mathrm{~nm}$, respectively. The diffracted wavelengths passes throughout an outlet slit $3 \mathrm{~mm}$ wide and after getting off the monochromator, the superior orders of diffraction is eliminated by means of a band pass optical filters. Monochromatic light is then modulated using a high stability mechanical chopper (Stanford SR 540) that gives to the system the reference pulse signal that is feed into the lock-in amplifier (EGG 5110). The collimated and modulated light is focused using two quartz lens ( $\mathrm{f} 1=100 \mathrm{~mm}$ and $\mathrm{f} 2=150 \mathrm{~mm}$ ) and the 
beam is directed onto the photoacoustic cell, passing by a quartz window and heats the sample. The cell body has a very sensitive and capacitive microphone coupled in (Brüel \& Kjäer BK 2669). The photoacoustic signal is collected by the microphone and fed into the lock-in. A personal computer (PC) using a usual IEEE boarding performs the signal detection and wavelengths scanning. The PAS acquisitions were all done using the frequency of $20 \mathrm{~Hz}$ and the light power was $800 \mathrm{~W}$.

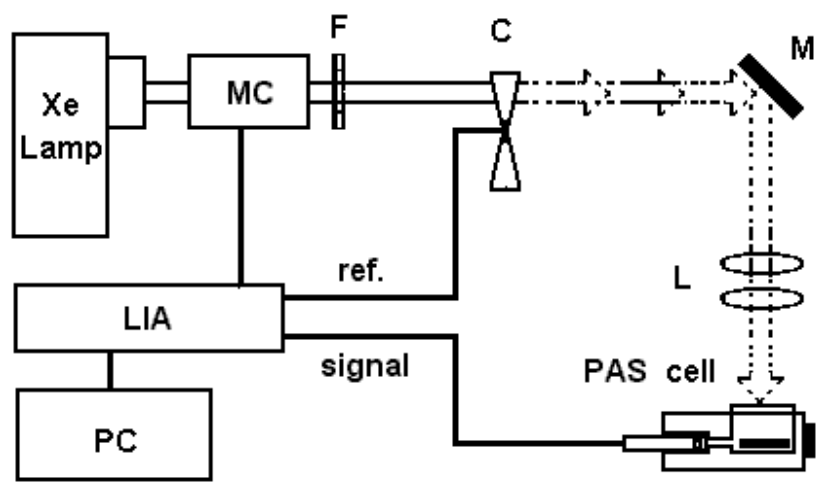

Figure 2. Photoacoustic spectrometer arrangement. $\mathrm{MC}=$ monochromator; $\mathrm{F}$ filter selecting band; $\mathrm{M}=$ mirror; $\mathrm{L}=$ lens; $\mathrm{C}=$ chopper; LIA lock-in-amplifier; $\mathrm{PC}=$ computer for data acquisition.

\section{Results and Discussion}

As we already have discussed earlier, the usage of the PAS was really necessary in order to follow the overtones of the absorption bands referring to hydroxyl bonds and their combinations with stretching modes that were seen presented in the spectral region of NIR and MIR. This was an efficient way for studying the raising of the cross-linking.

The total PAS spectra (NIR+MIR) for a specific sample grafted polyethylene with VTS (coded as $P E_{g} 7 \% 80^{\circ} \mathrm{C}$ ) can be observed in Fig. 3.

This is a representative spectrum observed for both set of samples, grafted PE and copolymer EVS. The whole set of PAS spectra is not shown but the same absorption structure was observed, despite of existing small differences in the intensities. Furthermore, no such absorption band could be related straightforward to the silanes group in the spectral range used. The observation of these groups could be very helpful as they indicate directly the cross-linking of the material. FTIR experiments could only show that silanes group was present and so, that the cross-linking process was initiated, but they were not able to show any evolution that would help us in this study.

The bands assignments for the samples used here were done by comparing the found absorption peaks with some preliminary studies in the polyethylene itself $[12,13]$. that have presented the assignment for almost absorption bands observed in our samples [19, 20].

We analyzed our PAS spectra and by comparing them we have assigned the polyethylene. Table I summarizes our findings and in this table the column named "peaks" are the labeled peaks presented in Fig. 3.

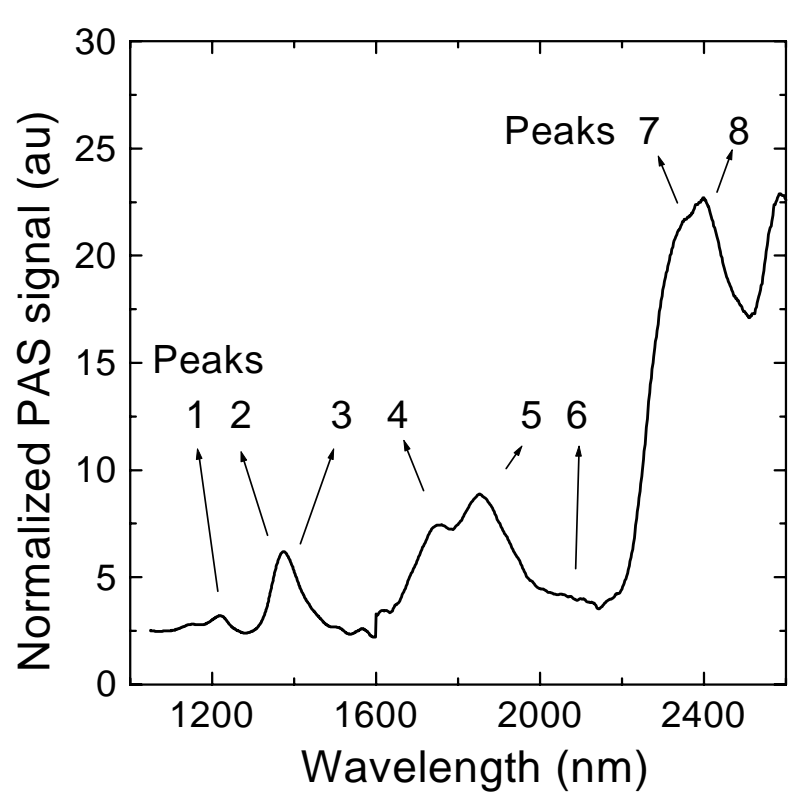

Figure 3. Typical photoacoustic spectrum for the $P E_{g} 7 \% 80^{\circ} \mathrm{C}$ sample given against wavelength in $\mathrm{nm}$.

The way we took for analyzing the PAS spectra was done performing a gaussian fitting of the spectra by setting the eight most prominent peaks observed in Fig. 3. The analysis strategy was to take the ratio of intensities for two distinct peaks for a sample, performing normalization with the same ratio of its respective "base". In this way one can observes if the cross-linking increases or decreases this "ratio". The following equation was used in this analysis [21]:

$$
\operatorname{ratio}(i, j)=\frac{\left(\text { peak }_{i} / \text { peak }_{j}\right)_{\text {sample\% }}}{\left(\text { peak }_{i} / \text { peak }_{j}\right)_{\text {base } \%}}
$$

By returning to Fig. 1(a) and Fig. 1(b) which shows the condensation reaction involved in the cross-linking process for $\mathrm{PE}_{g}$ and Cop, one can conclude that sample presenting PAS spectra with reduced intensity ratio for -OH groups, based upon Eq. (5), indicated that more cross-links are formed once the silane groups are crosslinked by means of the hydroxyl radical.

Following this statements we have plotted all ratios of the sample in Figs. 4 (NIR) and 5 (MIR). We advise the reader that these two figures are only representatives for the PAS intensities ratio, vertical axis. The horizontal axis gives us only a better visual sight of these ratios. 
Table I - Tabulated infrared bands assigned to PE in NIR and MIR range.[19]

\begin{tabular}{|c|c|c|}
\hline $\begin{array}{l}\text { Wavelength } \\
\text { nm }\end{array}$ & $\begin{array}{l}\text { Observed } \\
\text { Peaks }\end{array}$ & $\begin{array}{l}\text { Assignment } \\
\text { [observation] }\end{array}$ \\
\hline 1250 & 1 & 2nd overtone [characteristic of -CH2- and CH3- groups] \\
\hline 1400 & 2 & Free $O H, 1$ st overtone \\
\hline 1420 & 3 & Combination $\nu_{C H}{ }^{*}+\left[-\mathrm{CH}_{2}-, \mathrm{CH}_{3}-\right.$ groups $]$ \\
\hline 1760 & 4 & 1st overtone [characteristic of $-\mathrm{CH}_{2}-$ groups] \\
\hline $1800-1920$ & 5 & Free $O H$, combination \\
\hline 2020 & 6 & $\begin{array}{l}\text { Probably a combination [characteristic of terminal olefin methylene } \\
\text { group] }\end{array}$ \\
\hline $2080-2140$ & 6 & $\begin{array}{l}\text { Combination } \nu_{C H}{ }^{*}+[\text { characteristic of terminal olefin methylene group } \\
O-C H=C H 2 \text {. Region of } \nu_{O H} \text { combination band of alcohols] }\end{array}$ \\
\hline $2150-2200$ & 6 & Combination $\nu_{C H}+[$ characteristic of (cis) internal instauration $]$ \\
\hline 2240 & 7 & Combination $\nu_{C H}+\left[\right.$ characteristic of $\mathrm{CH}_{3}-$ groups $]$ \\
\hline $2300-2480$ & 7 and 8 & Combination $\nu_{\mathrm{CH}}+$ [characteristic of $-\mathrm{CH}_{2}-$ groups] \\
\hline
\end{tabular}

${ }^{*} \nu_{C H}$ stands for all possible combination modes of $\mathrm{CH}$ bonds, including symmetric and asymmetric vibration, stretching, rotation, torsion.
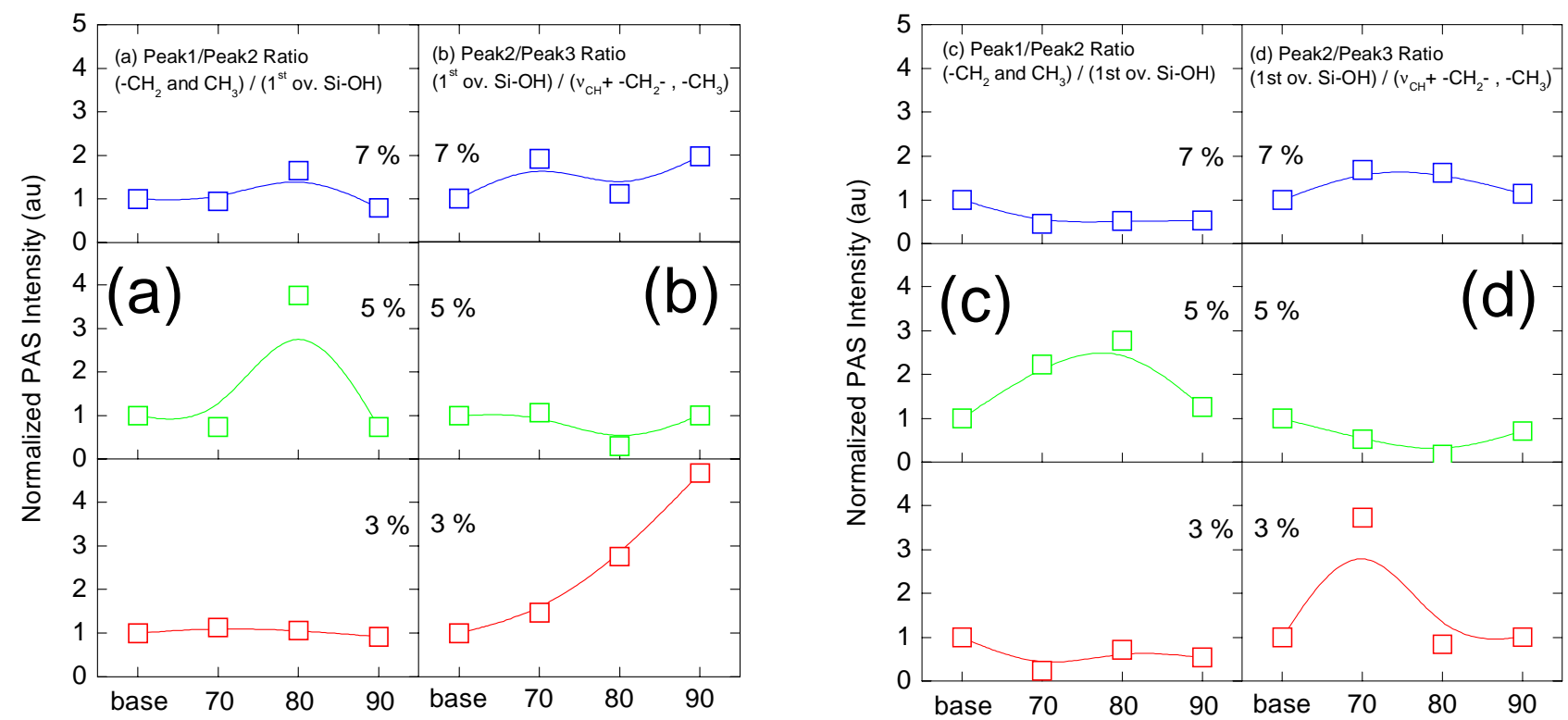

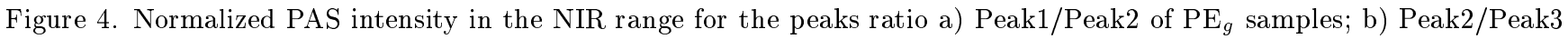
of $\mathrm{PE}_{g}$, c) Peak1/Peak2 of copolymer EVS samples; d) Peak2/Peak3 of copolymer EVS samples. Lines are eye guides.

Looking at the normalized peak ratios plotted in Fig. 4(a)-4(b) $\left(\mathrm{PE}_{g}\right)$ and 4(c)-(d) (Cop), referring to the NIR PAS spectra, we can see that between the $\mathrm{PE}_{g}$ and Cop sample with $5 \%$ catalyst and cross-linked at $80^{\circ} \mathrm{C}$ presented a better cross-linking rate. The ratio peak1/peak2, shown in Fig. 4(a) and 4(c) (frames for curves $5 \%$ ), were the most prominent and as a consequence, the ratio peak2/peak3, Fig. 4(b) and 4(d) (frames for curves 5\%), presented a small decreases. The latter, that is referred to the ratio [ $1^{\text {st }}$ overtone of $\left.\mathrm{Si}-\mathrm{OH} / v_{\mathrm{CH}}+-\mathrm{CH}_{2^{-}},-\mathrm{CH}_{3}\right]$ also indicates that the combination group's contribution to PAS intensity could be taking place. As we already have stated earlier, this ratios mainly shows that either PAS intensities assigned for $\mathrm{Si}-\mathrm{OH}$ have decreased or the PAS ratio for $-\mathrm{CH}_{2^{-}},-\mathrm{CH}_{3}$ and stretching combination $v_{C H}$ with the $-\mathrm{CH}_{2}$ - and $-\mathrm{CH}_{3}$ groups, have been enhanced. For instance, this result at least shows that samples $P E_{g} 5 \% 80^{\circ} \mathrm{C}$ and $C o p 5 \% 80^{\circ} \mathrm{C}$ were the best cross-linked ones. Still observing Fig. 4(a), sample $P E_{g} 7 \% 80^{\circ} \mathrm{C}$ have been also cross-linked but less than 
$P E_{q} 5 \% 80^{\circ} \mathrm{C}$ while samples $\operatorname{Cop} 7 \%$ (Fig. $4(\mathrm{c})$ ) have not change et all. On the other hand, the poorest crosslinked set looked to be the $3 \%$ catalyst samples. For both $\mathrm{PE}_{g}$ and Cop in Fig. 4(a) and 4(c), almost no changes can be seen in the frames for $3 \%$, the ratio [2 $2^{\text {nd }}$ overtones of $-\mathrm{CH}_{2}-$ and $\mathrm{CH}_{3^{-}} / 1^{\text {st }}$ overtone of $\mathrm{Si}-\mathrm{OH}]$ is constant but, in Fig. 4(b) and 4(d), there exist an indicator of a poor cross-linking process for the whole set of $7 \%$ and $3 \%$ catalyst, pointing the poorest as the $P E_{g} 3 \%$ set, Fig. $4(\mathrm{~b})$.

Figure 5 shows the PAS intensities for normalized peak ratios in the MIR region, where Fig. 5(a)-5(b) are for $\mathrm{PE}_{g}$ samples and Fig. 5(c)-(d) for Cop samples. In this figure we are plotting the normalized peak ratios for PAS absorption band assigned for $1^{\text {st }}$ overtone of $-\mathrm{CH}_{2}$, combination of free - $\mathrm{OH}$ and combination of $v_{\mathrm{CH}}$ with $-\mathrm{CH}_{2}-$. According to our strategy of analysis we conclude that sample $P E_{g} 5 \% 80^{\circ} \mathrm{C}$ seems to be the best cross-linked in this spectral range, Fig. 5 (a) frame named $5 \%$. By analyzing the plot ratios for $P E_{g} 3 \%$ and $P E_{g} 7 \%$ we can see a decreases in both plots, indicating a poor cross-linking reaction (see Fig. 5(a) frames 3\% and 7\%). If we take a look in Fig. 5(c) and (d) it is possible to see some degree of cross-linkings for samples $\operatorname{Cop} 3 \% 80^{\circ} \mathrm{C}, \operatorname{Cop} 3 \% 90^{\circ} \mathrm{C}, \operatorname{Cop} 5 \% 80^{\circ} \mathrm{C}$ and also for $\operatorname{Cop} 7 \% 70^{\circ} \mathrm{C}$. Although the increases in these normalized plot ratios for the MIR range show almost the same ratio, in general they were about 1.2, the NIR range shows a better result, where the ratio were near to 4.0 for $P E_{g} 5 \% 80^{\circ} \mathrm{C}$ and almost 3.0 for $C o p 5 \% 80^{\circ} \mathrm{C}$.

The overall of these results shows that by following $1^{\text {st }}$ overtone of free $\mathrm{OH}$ and combination of stretching frequencies for $-\mathrm{CH}_{2}-$ and $-\mathrm{CH}_{3}$ groups in the NIR, and also the $1^{\text {st }}$ overtone of $-\mathrm{CH}_{2}-$ and combination frequencies of free $O H$ in the MIR range, it is possible to have some insights about the cross-linking process in these two polymer, grafted PE with VTS and EVS copolymer. Mainly it was observed that $80^{\circ} \mathrm{C}$ was enough to have a good cross-linking when it is combined with $5 \%$ of catalyst.

Owing to monitor the cross-linking effects that would be present in the sample, we have also performed a Frequency Scanning in the light pulse at some specific wavelengths. The range of this frequency scanning was 10 to $100 \mathrm{~Hz}$ at the wavelengths $1732 \mathrm{~nm}$ (peak4 assigned as $1^{\text {st }}$ overtone of $-\mathrm{CH}_{2}-$ groupings), $1850 \mathrm{~nm}$ (peak5 attributed to free $O H$ combination), $2300 \mathrm{~nm}$ (peak7 combination of $-\mathrm{CH}_{2}-$ groups) and $2400 \mathrm{~nm}$ (peak8 also $-\mathrm{CH}_{2}-$ combination). In general, in doing such scanning one may be able to make studies of depth profiles distribution for the absorption groups beneath the surface of the polymer. This information is also very important because the cross-linking activation is believed to start at the polymer surface. Our findings in studying both silane grafted $\mathrm{PE}\left(\mathrm{PE}_{g}\right)$ and copolymer EVS (Cop) are discussed below.
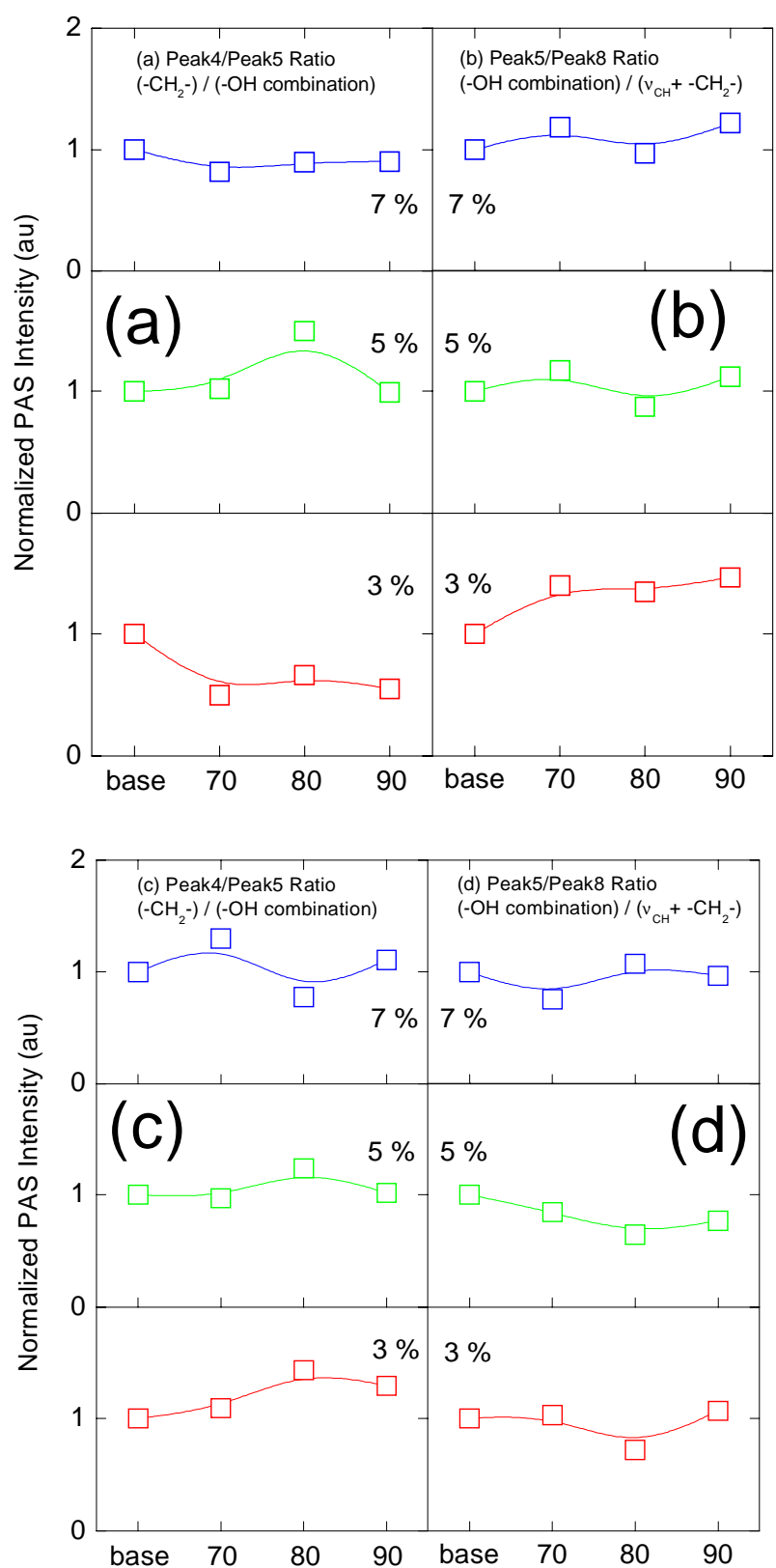

Figure 5. Normalized PAS intensity in the MIR range for the peaks ratio a) Peak4/Peak5 of samples $\mathrm{PE}_{g}$; b) Peak5/Peak8 of $\mathrm{PE}_{g}$; c) Peak4/Peak5 of copolymer EVS samples; d) Peak5/Peak8 of copolymer EVS samples. Lines are eye guides.

By fitting the log-log plots of PAS intensity against frequency of pulse for all representative peaks described in the last paragraph, we observed that the absorption band intensities showed a negative slop, varying as $\omega^{-a}$, where $\omega$ is the angular modulation frequency in Hertz and the fitted parameter "a" is the slope. It was observed "a" from 0.9 to 1.2 for the peaks from $\mathrm{PE}_{g}$ samples and it runs from 1.0 to 1.4 for the Cop samples.

According to the photoacoustic general theory the modulation of the frequency can be used as a probe by means of the expression $\mu(\omega)=(2 \alpha / \omega)^{1 / 2}$. Here, $\alpha$ is the polymer bulk thermal diffusivity, $\mu$ is the heat 
depth penetration at $\omega$. It means that $\mu$ is ruled by the frequency $\omega$. So, at lower frequencies the photoacoustic intensity is mainly produced by the polymer bulk absorption and otherwise, at higher frequencies, the intensity is due to polymer surface absorbing groups.

In a previous study in impregnated LDPE, Ganzarolli et al. [19] have proposed that if the slope "a" is less then $(-3 / 2)$ one can assume that the absorption groups that is generating the PAS signal may be more concentrated in the polymer surface rather than in its bulk. The consequence of this is that sample might have a non-uniform thermal diffusivity $\alpha$. In average, the frequency scanning at peaks assigned to $1^{\text {st }}$ overtone of $-\mathrm{CH}_{2}-$ and to free $\mathrm{OH}$ combination, peak4 and 5 , have not shown so much differences for the $\mathrm{PE}_{g}$ series. The PAS intensity slope were found $S_{f} \sim \omega^{-0.8}$ and $S_{f} \sim \omega^{-0.9}$, respectively, while it were observed $S_{f} \sim \omega^{-1.3}$ for both peak7 and peak8.

Now as to compare, in the series Cop on the other hand, the PAS intensity slope were observed to be different for peak $4, S_{f} \sim \omega^{-1.2}$, and peak $5, S_{f} \sim \omega^{-1.0}$, respectively. For peaks 7 and 8 the PAS intensity slope were almost not distinguished, remained in the range $S_{f} \sim \omega^{-1.4}$ to $S_{f} \sim \omega^{-1.5}$. Although the peak intensity slopes have not shown any distinct cross-linking effect for both series, $\mathrm{PE}_{g}$ and Cop, by using frequencies scanning we conclude that groupings $-O H$ (peak5) and $-\mathrm{CH}_{2}-$ (peak7) have greater concentration in the polymer surface rather than in their bulks. Also this gradient pattern is greater for $-\mathrm{CH}_{2}-$ than $-\mathrm{OH}$ grouping. Another conclusion we can retrieve from intensities slopes is that grafted PE samples present these absorbing groups more concentrated at the surface when compared to slopes found for Cop samples. This conclusion was supported by analyzing the whole PAS spectra at 20,40, 50 and $80 \mathrm{~Hz}$, in the range 1600 $\mathrm{nm}$ to $2600 \mathrm{~nm}$ for samples $\mathrm{PE}_{g}$, it was observed that the peaks assigned for $-\mathrm{CH}_{2}-$ and $-\mathrm{OH}$ decreases at higher frequencies, but the decreases is more pronounced for $-\mathrm{CH}_{2}-$ groups (by comparing PAS spectra at 20 and $80 \mathrm{~Hz}$, respectively). That means that while intensity for $-\mathrm{CH}_{2}$ - is fading way, the intensity for $-O H$ still remains at higher frequencies.

In table II it is summarized the calculation of the gel content and crystalline percentage. Gel content is determined by weighting the cross-linked polymer before and after it has been exposed to an appropriated solvent. The cross-linked phase has a very low solubility when exposed to the solvent presenting intumescences [22]. The degree of crystallinity of this phase is then determined by x-ray diffraction. The crystalline fraction was obtained by integrating the peak area from two peaks, one for assigned crystalline plane [110] at $2 \theta \sim 21.60$ and the other for plane [200] at $2 \theta \sim 24.20$. The amorphous peak was integrated with the center at $2 \theta \sim 20.10[23,24]$.

Table II - Summary of apparent gel content and crystallinity.

\begin{tabular}{ccc}
\hline \hline Samples Coding & $\begin{array}{c}\text { Apparent gel content } \\
(\%)\end{array}$ & $\begin{array}{c}\text { Crystallinity from x-ray } \\
(\%)\end{array}$ \\
\hline$P E_{g} 3 \% 70^{\circ} \mathrm{C}$ & 32.7 & 70.1 \\
$P E_{g} 3 \% 80^{\circ} \mathrm{C}$ & 35.8 & 43.6 \\
$P E_{g} 3 \% 90^{\circ} \mathrm{C}$ & 44.7 & 37.9 \\
$P E_{g} 5 \% 70^{\circ} \mathrm{C}$ & 36.9 & 63.5 \\
$P E_{g} 5 \% 80^{\circ} \mathrm{C}$ & 37.5 & 60.1 \\
$P E_{g} 5 \% 90^{\circ} \mathrm{C}$ & 45.6 & 49.9 \\
$P E_{g} 7 \% 70^{\circ} \mathrm{C}$ & 45.2 & 65.4 \\
$P E_{g} 7 \% 80^{\circ} \mathrm{C}$ & 47.2 & 64.7 \\
$P E_{g} 7 \% 90^{\circ} \mathrm{C}$ & 49.3 & 48.8 \\
$C o p 3 \% 70^{\circ} \mathrm{C}$ & 60.2 & 27.4 \\
$C o p 3 \% 80^{\circ} \mathrm{C}$ & 55.2 & 71.0 \\
$C o p 3 \% 90^{\circ} \mathrm{C}$ & 65.5 & 43.1 \\
Cop $5 \% 70^{\circ} \mathrm{C}$ & 67.1 & 62.2 \\
Cop $5 \% 80^{\circ} \mathrm{C}$ & 69.6 & 59.0 \\
Cop $5 \% 90^{\circ} \mathrm{C}$ & 74.9 & 40.7 \\
Cop $7 \% 70^{\circ} \mathrm{C}$ & 69.9 & 47.4 \\
Cop $7 \% 80^{\circ} \mathrm{C}$ & 74.4 & 36.7 \\
Cop $7 \% 90^{\circ} \mathrm{C}$ & 74.6 & 60.9 \\
\hline
\end{tabular}


It can be observed an apparent increasing in the gel content with temperature for all catalyst concentration. The $P E_{g} 5 \% 80^{\circ} \mathrm{C}$ and $P E_{g} 5 \% 90^{\circ} \mathrm{C}$ present nearly the same value of $P E_{g} 7 \%$. Also it can be seen that $\mathrm{PE}_{g}$ samples present lower gel content then that for Cop. This result is believed to be due to a peculiar increasing of the cross-linkings once the photoacoustic frequency scanning results indicate that $\mathrm{PE}_{g}$ has a more remarkable cross-linking effects in the surface whereas in the Cop, it looked to spread out in the bulk. In the analysis of crystallinity, one can see that it decreases with temperature and it is follows that it indicates a larger degree for $\mathrm{PE}_{g}$ than that for Cop samples, if similar samples are to be compared. The majority of samples in the set have pointed this way.

The conclusion one can take from the observed effects is that if sample is cross-linked at higher temperature, the cross-linking degree is enhanced and on an opposite way, the degree of crystallinity decreases. It appears that there is a competition between both and is reasonable to say that $80^{\circ} \mathrm{C}$ appears to be the optimized point for sample preparation.

\section{Conclusion}

The PAS has pointed out that in the set of sample we used, the better variable for cross-linking was $80^{\circ} \mathrm{C}$ and catalyst in the range 5 to $7 \%$, typically. It was also shown that PAS is a capable technique for evaluating cross-linking rate in copolymer and grafted PE, by analyzing the overtones absorption band in the NIR and MIR. In the frequency scanning analysis it was observed no distinct cross-linking effect for both series, $\mathrm{PE}_{g}$ and Cop, but it locates groupings $-\mathrm{OH}$ (peak5) and $-\mathrm{CH}_{2}-$ (peak7) more concentrated in the surface, showing a larger gradient for $-\mathrm{CH}_{2}-$ than $-\mathrm{OH}$. Intensities slopes indicate that grafted $\mathrm{PE}$ present these groups more concentrated at the surface when compared to those for Cop samples.

\section{Acknowledgments}

The authors acknowledge the Brazilian agencies $\mathrm{CNPq}$ and Capes for the partial support of this work.

\section{References}

[1] A. Rosencwaig, Photoacoustic and Photoacoustic Spectroscopy, (John Wiley \& Sons, New York, 1980).

[2] D. P. Almond and P. M. Patel, Photothermal Science and Technology, (Chapman \& Hall, London, UK, 1996).

[3] H. Vargas and L. C. M. Miranda, Phys. Rep. 161, 43 (1988).
[4] 11th ICPPP - International Conference on Photoacoustic and Photothermal Phenomena, Book of abstracts, ed. T. Sawada, 25-29 June/2000, Kyoto, Japan, collection of papers therein.

[5] S.Grzybowski, P. Zubielik, and E. Kuffel, IEEE Transactions on Power Delivery 4, 1507 (1989).

[6] H. G. Elias, in Macromolecules 1 Strutucture and Proprieties, $2^{\text {nd }}$ ed., (Plenum Press, New York, 1983).

[7] M. Markis, A. Tzur, A. Vaxman, Polym. Eng. Sci. 25, 13 (1985).

[8] K. B. Whetsel, Appl. Spec. Rev. 2, 1 (1968).

[9] L. G. Weyer, Appl. Spec. Rev. 21, 1 (1985). [Inner references: S. Glasstone, Theoretical Chemistry. An Introduction to Quantum Mechanics, Statistical Mechanics, and Molecular Spectra for Chemists, Van Nostrand, New York, 180 (1944)].

[10] I. A. Cowe, Near Infrared Analysis - Today or Tomorrow?, Anal. Proc. (London), 20, 65 (1983).

[11] S. Cartasegna, Rubber Chem. and Technol. 59, 722 (1986).

[12] A. Kumar Sen, B. Mukherjee, A. S. Bhattacharyya, P. P. De, and A. K. Bhowmick, J. Appl. Polym. Sci. 44, 1153 (1992).

[13] T. Hjertberg, M. Palmlof, and B. A. Sultan, J. Appl. Polym. Sci. 42, 1185 (1991).

[14] M. Palmlöf, T. Hjertberg, and B. A. Sultan, J. Appl. Polym. Sci. 42, 1193 (1991).

[15] A. K. Sem, B. Mukherjee, A. S. Bhattacharyya, P. P. De, and A. K. Bhowmick, J. Therm. Anal. 39, 887 (1993).

[16] A. J. Muller, Z. H. Hernandez, M. L. Arnal, and J. J. Sánchez, Polym. Bullet. 39, 465 (1997).

[17] W. K. Wong, D. C. Varrall, Polym. 35, 5447 (1994).

[18] I. L. Hosier, A. S. Vaughan, and S. G. Swingler, J. Mat. Sci. 32, 4523 (1997).

[19] M. G. Oliveira, O. Pessoa Jr., H. Vargas, and F. Galembeck, J. Appl. Polym. Sci. 35, 1791 (1988).

[20] L. Glatt and J. W. Ellis, J. Chem. Phys. 19, 449 (1951).

[21] D. T. Dias, Master Dissertation presented to Departamento de Física, Universidade Estadual de Maringá, Paraná, Brazil, (2001).

[22] M. F. Porto, Master Dissertation presented to Programa de Mestrado em Química Aplicada, Universidade Estadual de Maringá-UEM, (2001).

[23] B. E. Warren, X-ray Diffraction, Addison-Wesley, London, pp.13-16 (1969).

[24] S. L. Aggarwal and G. P. Tilley, J. Polym. Sci. XVIII, 15-25, (1955). 